\title{
Some Remarks on Local Operators in Quantum Electrodynamics*
}

\author{
R. Ferrari and L. E. Picasso \\ Istıtuto dı Fisica dell'Universitá, Pisa, Istituto Nazionale de Fisica Nucleare, Sezione di Pisa, \\ Pisa, Italy \\ F. Strocchi ${ }^{\star \star}$ \\ Joseph Henry Laboratories of Physics, Princeton University, Princeton, New Jersey, USA
}

Received September 25, 1972; in revised form June 17, 1973

\begin{abstract}
We assume the existence of a conserved current which generates locally gauge transformations of first kind. We are working in a local quantum Field Theory, where the fields are defined on a vector space where indefinite metric is allowed.

We show that the Maxwell equations are not consistent with the above assumptions and the vectors obtained by applying local charged operators on the vacuum cannot describe physical states.

Moreover we show that, if charged fields have non-trivial expectation value on the physical states, the vector space must contain vectors with negative norm.

We discuss the relation between the local formulation of QED and a formulation in terms of physical states. As an example we study the transition from Gupta-Bleuler free QED to the Coulomb-gauge formulation.
\end{abstract}

\section{Introduction}

The aim of the present note is to discuss locality in quantum electrodynamics (QED) and in particular to analyze the restrictions imposed by this property on the structure of the theory. A way to get information about the formal structure of the theory is to use perturbation theory. In that way, the general properties like locality and covariance are essentially dictated by the free field case and one is essentially led to the standard formulations (the local and manifestly covariant GuptaBleuler formulation [1] or the non local and non manifestly covariant Coulomb gauge formulation [2]). However, since very little is known about the convergence of the renormalized perturbation expansion, it is of some interest to discuss some general features of the theory, in

* Research supported by AFOSR, Contract F 44620-71-C-0108

$\star \star$ Present address: Istituto Nazionale di Fisica Nucleare, Sezione di Pisa, Pisa, Italy. 
particular locality, in the framework of Wightman theory [3], with no reference to the lagrangian formalism. In this way one may get information about formulations which may be more general than that of the usual lagrangian approach. The main point of this note is to show that actually the characteristic features of the standard (Gupta-Bleuler and Coulomb gauge) formulations are much more general than one might think and are strongly connected with the property of locality. As we will show, one cannot hope to formulate QED in term of $F_{\mu \nu}, j_{\mu}$ and local charged fields without essentially going to the Gupta-Bleuler formulation. Fields describing charged particles can be defined as local fields only in a Hilbert space equipped with an indefinite metric and only if the Maxwell's equations are abandoned as operator equations. This result will be obtained without ever introducing the electromagnetic potential $A_{\mu}$, and therefore no reference is made to particular gauge conditions. As a consequence of the above result we will see that in any formulation of the Gupta-Bleuler type, the algebra $\mathscr{R}$ of local operators leaving the subspace $H^{\prime}$ invariant, commutes with the automorphism induced by the electromagnetic current and therefore it cannot contain charged field operators. (For a more detailed and precise discussion see Section 3.)

In this note we are explicitly concerned with interacting QED and the present results can be considered as the analogous for the interacting case of the already established results in free QED [4].

\section{Locality and Maxwell's Equations}

In this section we will discuss the property of locality in connection with the Maxwell's equations. Since no reference is made to the lagrangian formulation one has to specify some of the basic properties of $j_{\mu}$ and $F_{\mu v}$ in order to properly pose the problem. We therefore assume that the fields $j_{\mu}(x)$ and $F_{\mu \nu}(x)$ can be defined as operator valued distributions in a Hilbert space $G$. Since we want to identify $j_{\mu}$ with the electromagnetic current, we have to specify some other property of this field. For example if $j_{\mu}=m^{2} A_{\mu}$, the equations $\partial_{\mu} F^{\mu v}=j^{v}$ would describe a free massive vector field $A_{\mu}$, with no connection to QED.

A necessary condition to be satisfied by $j_{\mu}$ in order to be a candidate for the electromagnetic current is that $\partial_{\mu} j^{\mu}(x)=0$ and that the integral of the zero-th component of $j_{\mu}$ is associated to the electric charge. The above statement may be made precise in the following way.

Let us define

$$
Q_{R, d} \equiv \int j^{0}\left(\boldsymbol{x}, x_{0}\right) f_{\boldsymbol{R}}(\boldsymbol{x}) f_{d}\left(x_{0}\right) d^{3} x d x_{0}
$$


where

$$
\begin{aligned}
& f_{R}(\boldsymbol{x})=1 \quad \text { for } \quad|\boldsymbol{x}|<R, \\
& f_{R}(\boldsymbol{x})=0 \text { for } \quad|\boldsymbol{x}|>R+\varepsilon, \\
& f_{d}\left(x_{0}\right)=0 \text { for } \quad\left|x_{0}\right|>d, \\
& \int_{-\infty}^{+\infty} f_{d}\left(x_{0}\right) d x_{0}=1 .
\end{aligned}
$$

Then for any local field $\phi(x)$ and for any test function $f$ of compact support the $\lim _{R \rightarrow \infty}\left[Q_{R}, \phi(f)\right]$ exists $^{1}$. We may therefore define

Definition. A field $\phi(x)$, local relative to $j_{\mu}(x)$ is said to have charge $q$ if for any $f \in \mathscr{D}\left(R^{4}\right)$

$$
\lim _{R \rightarrow \infty}\left[Q_{R}, \phi(f)\right]=-q \phi(f)
$$

Up to now the definitions were quite general and they do apply to any conserved current $j_{\mu}$. The basic assumption which allow us to identify $j_{\mu}$ with the electromagnetic current is that the "charge" occurring in Definition 1 is the electric charge, in the sense that it generates locally the group of automorphisms $U(1)$ corresponding to gauge transformations of the first kind.

Lemma $1^{2}$. If $j_{\mu}(x)$ is a local current of the form $j_{\mu}(x)=\partial^{v} F_{v \mu}(x)$, where $F_{\mu \nu}(x)=-F_{v \mu}(x)$ is a local field, then for any field $\phi(x)$ local relative to $j_{\mu}$ and $F_{\mu \nu}$ one has

$$
\lim _{R \rightarrow \infty}\left[Q_{R}, \phi(f)\right]=0 .
$$

Proof. We consider the commutator

$$
\left[Q_{R}, \phi(f)\right] \text {. }
$$

The current $j_{\mu}$ is conserved since $F_{\mu \nu}$ is antisymmetric:

$$
\partial^{\mu} j_{\mu}=\partial^{\mu} \partial^{v} F_{\mu v}=0 \text {. }
$$

Then, according to well known arguments [5], the above commutator is independent of $f_{R}$ and $f_{d}$ provided that $\operatorname{supp} f$ is contained in the dependence domain of the surface $\left\{x \in R^{4}: x_{0}=0,|x|<R-d\right\}$. As a matter of fact in this case the only points in the integral $\int j^{0} f_{R} f_{d} d^{4} x$, which contribute to the commutator, are those with $|x|<R-d$.

\footnotetext{
1 From now on we will omit the subscript $d$ in $Q_{R, d}$, since the conservation of $j_{\mu}$ implies that $\left[Q_{R, d}, \phi(f)\right]$ is independent of $f_{d}$ for $R$ sufficiently large.

2 After this work was completed we were informed by Professor R. Haag that a similar statement was made by J. Swieca (unpublished).
} 
Therefore, from the hypotheses, one has

$$
\begin{aligned}
{\left[Q_{R}, \phi(f)\right] } & =\int f_{R}(\boldsymbol{x}) f_{d}\left(x_{0}\right)\left[j^{0}\left(\boldsymbol{x}, x_{0}\right), \phi(f)\right] d^{4} x \\
& =\int f_{R} f_{d}\left[\partial_{\mu} F^{\mu 0}(x), \phi(f)\right] d^{4} x \\
& =\int f_{R} f_{d}\left[\partial_{i} F^{i 0}(x), \phi(f)\right] d^{4} x \\
& =-\left[F^{i 0}\left(\partial_{i} f_{R}, f_{d}\right), \phi(f)\right], \quad i=1,2,3 .
\end{aligned}
$$

But $f_{R}(\boldsymbol{x})$ is constant for $|\boldsymbol{x}| \leqq R$, i.e. on the region of points which can contribute to the above commutator. Hence one has

$$
\lim _{R \rightarrow \infty}\left[Q_{R}, \phi(f)\right]=0 .
$$

Theorem 1. In any local quantum field theory in which $j_{\mu}$ generates non-trivial automorphism on the local algebra the Maxwell equations

cannot be valid.

$$
j_{\mu}=\partial^{v} F_{v \mu}
$$

This follows from Lemma 1.

Remarks. a) No assumption has been made about the existence of a field $A_{\mu}(x)$ such that $F_{\mu \nu}=\partial_{\mu} A_{\nu}-\partial_{\nu} A_{\mu}$. The conclusion of the above theorem applies also to formulations in which $A_{\mu}$ is never introduced.

b) As a consequence of the above theorem one cannot hope to formulate QED in terms of only $F_{\mu \nu}, j_{\mu}$ and the charged fields, if these are local fields ${ }^{3}$. An unphysical local field $\mathscr{A}^{v}(x)=\partial_{\mu} F^{\mu v}(x)-j^{v}(x)$ must necessarily be introduced. In the standard Gupta-Bleuler formulation one has $\mathscr{A}^{v}(x)=-\partial^{v} \partial_{\mu} A^{\mu}(x), A_{\mu}$ being the vector potential.

c) Theorem 1 shows that in any theory in which $\partial_{\mu} F^{\mu \nu}=j^{v}$, like in the Coulomb gauge formulation of QED, the charged fields cannot be defined as local fields. This result, known for the Coulomb gauge, is thus shown to have a general validity.

\section{Properties of a Local Formulation of QED}

In order to get further insight on the structure of a possible local formulation of QED we need some notations and definitions. We start by stating the basic assumptions which give a precise definition of a local formulation of QED.

\footnotetext{
${ }^{3}$ Here and in the following we discuss only the case of local fields. The results of Theorem 1 and most of the results of this note remain true for quasi local fields.
} 
1) The fields are defined as operator valued distributions of type $\mathscr{S}^{\prime}$ or $\mathscr{D}^{\prime}$ in a Hilbert space $G$. $G$ is equipped with a sesquilinear hermitean form $\eta=\eta^{*}$ (* denotes the Hilbert space adjoint), in terms of which all the physically meaningful quantities like transition probabilities, vacuum expectation values, etc. are computed and the representation at the Poincare group is unitary. That means that besides the ordinary scalar product $($,$) in G$, one has the following "product"

$$
\langle\Psi, \Phi\rangle_{\eta} \equiv\langle\Psi, \Phi\rangle=(\Psi, \eta \Phi) \text {. }
$$

We do not make any assumption about $\eta$ (it could even turn out that one may choose $\eta=1$ ), except that $\eta$ is non degenerate, i.e. that there is no subspace $G_{0} \subset G$ such that

$$
\eta \Psi=0, \quad \forall \Psi \in G_{0} .
$$

2) The algebra of local and covariant fields in $G$ is denoted by $\mathscr{I}$. As usual, we assume that $\mathscr{I}$ contains enough fields so that $\mathscr{I}$ is irreducible in $G$.

3) The basic assumption on $j_{\mu}$, given in Section 2 , may be now stated more precisely: $j_{\mu}$ generates the weakly continuous group $U(1)$ of automorphisms of the gauge transformations of the first kind on the local fields. These transformations commute with Poincaré's transformations.

As a consequence of Theorem 1 one cannot assume $\partial_{\mu} F^{\mu v}=j^{v}$ in $G$, if $\mathscr{I}$ contains charged fields as expected. Moreover $\mathscr{I}$ must contain unphysical fields like $\mathscr{A}^{v}(x)$. Clearly in order to have contact with physics one has to give a criterium to select those vectors of $G$ which are candidate to describe physical states. For them, Maxwell's equations should hold as mean values and they should have non zero $\eta$-norm. A more precise statement about the properties of those vectors is given by the following definition

Definition. $H^{\prime}$ is a linear manifold in $G$ such that

i) The domain $D \subset H^{\prime}$ on which the smeared fields $F_{\mu \nu}(f), j_{\mu}(f)$ can be defined and such that $F_{\mu \nu}(f) D \subset D, j_{\mu}(f) D \subset D$ is dense in $H^{\prime}$.

ii) Maxwell's equations hold as mean values on $H^{\prime}$ :

$$
\begin{gathered}
\left\langle\Psi,\left(\partial_{\mu} F^{\mu v}(f)-j^{v}(f)\right) \Phi\right\rangle=0, \\
\left\langle\Psi, \varepsilon_{\mu \nu \varrho \sigma} \partial^{v} F^{\varrho \sigma}(f) \Phi\right\rangle=0, \quad \forall \Psi \in H^{\prime}, \quad \forall \Phi \in D .
\end{gathered}
$$

iii) $\langle\Psi, \Psi\rangle \geqq 0, \quad \forall \Psi \in H^{\prime}$.

It is not difficult to recover the physical motivations for expecting that properties i), ii), iii) should hold for vectors of $G$ which are candidates to describe physical states. 
Theorem 2. Unless all the charged fields of $\mathscr{I}$ have zero expectation values between vectors of $H^{\prime}$

a) Maxwell's equations do not hold as operator equations on $D$ and there are vectors of $H^{\prime}$ with zero $\eta$-norm and

b) $\eta$ cannot be semidefinite in $G$, i.e. there must be vectors $\Psi$ in $G$ of negative $\eta$-norm: $\langle\Psi, \Psi\rangle<0$.

Proof. The proof simplifies if one makes the technical assumption that for the smeared charged fields $\phi(f)$, the bilinear form

$$
\left\langle\Psi,\left[\mathscr{A}_{R}, \phi(f)\right] \Phi\right\rangle
$$

is defined for any $\Psi, \Phi \in D$. (For a more general proof see Appendix.)

One first notices that, by Lemma $1, \mathscr{A}_{R}=\int \mathscr{A}^{0}(x) f_{R}(\boldsymbol{x}) f_{d}\left(x_{0}\right) d^{3} x d x_{0}$ and $Q_{R}$ induce the same automorphism on the fields of $\mathscr{I}$. Then, $\forall \Psi, \Phi \in D$ and for any charged field $\phi_{q} \in \mathscr{I}$, carrying charge $q$, one has

$$
\lim _{R \rightarrow \infty}\left\langle\Psi,\left[\mathscr{A}_{R}, \phi_{q}(f)\right] \Phi\right\rangle=-q\left\langle\Psi, \phi_{q}(f) \Phi\right\rangle .
$$

Hence, if $\mathscr{A}^{v}(f) D=0$ one gets

$$
q\left\langle\Psi, \phi_{q}(f) \Phi\right\rangle=0,
$$

i.e. either $q=0$ or $\left\langle\Psi, \phi_{q}(f) \Phi\right\rangle=0$.

This proves that a situation where charged fields have non-trivial expectation value on $D$ rules out the possibility that Maxwell's equations are valid in a subspace $H^{\prime} \subset G$, i.e. $\mathscr{A}^{v}(f) \Phi=0$ for any $\Phi \in D$. It is a direct consequence of property (ii) that if $\Psi=\mathscr{A}^{v}(f) \Phi \neq 0$ then

$$
\langle\Psi, \Psi\rangle=\left\langle\mathscr{A}^{\nu}(f) \Phi, \Psi\right\rangle=0,
$$

i.e. the vector space $H^{\prime \prime} \subset H^{\prime}$ of vectors with zero $\eta$-norm is not empty.

To prove b), let us suppose $\eta$ semipositive on $G$. Then, one may show that a vector of vanishing $\eta$-norm has zero $\eta$-products with any vector of $G$. This is in contrast with $\eta$ not being degenerate.

Remarks. The non-existence of $H^{\prime}$ or the vanishing of the expectation values of all the charged fields between vectors of $D$ would mean that one cannot hope to have a reasonable local formulation of QED. Therefore, we will confine our attention to theories in which $H^{\prime}$ is not empty and some of the charged fields of $\mathscr{I}$ have non-vanishing expectation values on $H^{\prime}$. In this case the main conclusion to be drawn from Theorem 2 is that one cannot hope to select physically relevant vectors of $G$ as in the Definition without necessarily picking up unphysical vectors of zero norm and without abandoning Maxwell's equations as operator equations on $D$. It may be worthwhile to remark that the possibility of having the Maxwell's equations satisfied in $D$ is not ruled out by 
Theorem 1 only, since the charged fields are not assumed to leave $D$ invariant (in fact they don't, see later).

In conclusion, in order to have local fields which do not commute with the automorphism induced by $Q_{R}$ one must define the theory in a Hilbert space equipped with indefinite metric, introduce unphysical fields $\left(\mathscr{A}^{v}(x)\right)$, unphysical vectors and vectors of negative $\eta$-norm. Thus, any local formulation of QED must share all the characteristic features of the Gupta-Bleuler formulation.

As it is well known [6], in the Gupta-Bleuler formulation of free QED the indefinite metric is introduced following different reasons than those stated in Theorem 2 . Here it is crucial to assume that not all charged fields have zero expectation value on $H^{\prime}$. This difference is clearly seen in our framework. If no physically relevant charged fields were present, our treatment would suit equally well Gupta-Bleuler or Coulomb formulation of QED, since $F_{\mu \nu}, j_{\mu}$ and neutral fields can be relatively local and allow the validity of Maxwell equations (see Theorem 1). Then there is no need of indefinite metric as in Coulomb formulation.

Corollary 1. Let $\phi_{q}(f)$ be a charged field with $f \in \mathscr{D}\left(R^{4}\right)$. Denote by $\tilde{D} \subset H^{\prime}$ the domain on which the bilinear form $\left\langle\Psi,\left[\mathscr{A}_{R}, \phi_{q}(f)\right] \Phi\right\rangle(R$ sufficiently large, $\Psi, \Phi \in \tilde{D})$ can be defined, then

$$
\phi_{q}(f) \tilde{D} \not \subset H^{\prime}
$$

unless $q\left\langle\Psi, \phi_{q}(f) \Phi\right\rangle=0, \forall \Psi, \Phi \in \tilde{D}$.

Proof. Let $\Psi, \Phi \in \tilde{D}$, then if $\phi_{q}(f) \tilde{D} \subset H^{\prime}$ one has $-q\left\langle\Psi, \phi_{q}(f) \Phi\right\rangle$ $=\left\langle\Psi,\left[\mathscr{A}_{R}, \phi_{q}(f)\right] \Phi\right\rangle$ for $R$ large enough. But $\mathscr{A}_{\mu}$ has zero expectation value between vectors of $H^{\prime}$ and therefore $q\left\langle\Psi, \phi_{q}(f) \Phi\right\rangle=0$.

It is important to stress that in the results obtained up to now the existence of a vacuum state (a non-trivial assumption in a theory with massless particles) and the spectral condition were not assumed. The content of Corollary 1 may be slightly strenghened under the assumption that there is a vacuum state $\Psi_{0}$, cyclic with respect to $\mathscr{I}$. In this case if $\mathscr{R}$ denotes the algebra of local operators which leave $H^{\prime}$ invariant, one may consider the subspace $D_{0}$ of local states of $H^{\prime}: D_{0} \equiv$ set of vectors of $H^{\prime}$ obtained by applying elements of $\mathscr{R}$ to the vacuum $\}$. Then Corollary 1 can be reformulated in the form

Corollary 2. Let $A$ be any element of $\mathscr{R}$, then either

a) $A$ has zero charge or

b) for any $\Phi \in H^{\prime}, \Psi \in D_{0}$

$$
(\Phi, A \Psi)=0
$$

and therefore $A \Psi \in H^{\prime \prime}$. 
Proof. One may show ${ }^{4}$ that $\mathscr{R}$ is generated by elements of definite charge $A_{q}$. Then Corollary 1 can be applied straightforwardly.

\section{Physical Interpretation of the Local Theory}

In order to get a physical interpretation out of the theory discussed above one has to give prescriptions as how to get rid of all the unphysical features such as unphysical fields, states of negative norm and states of zero norm. This may be done by introducing the quotient space $H=H^{\prime} / H^{\prime \prime}$ in which one has positive definite scalar product and in which Maxwell's equations hold as operator equations [7].

A question which has to be discussed is in which way operators in $G$ give rise to operators in $H$. To be more precise, if $O$ is a bounded operator defined in $G$, how may one define an operator $\hat{O}$ which acts on $H$ and may be interpreted as the operator corresponding to $O$ ? To discuss this problem we introduce the following notation: if $\Psi$ is a vector of $H^{\prime}, \hat{\Psi}$ will denote the equivalence class of $\Psi$ with respect to $H^{\prime \prime}$. Therefore $\hat{\Psi}$ may be regarded as a vector of $H^{5}$.

A mapping $O \rightarrow \hat{O}$ may be defined in the following way. One chooses an orthonormal basis $\hat{\Psi}_{i}$ in $H^{6}$, and for each equivalence class one picks up a vector $\Psi_{i}$ of $H^{\prime}, \Psi_{i} \in \hat{\Psi}_{i}$.

4 We give a brief sketch of the proof. Let $\alpha$ be an element of the group $U(1)$ introduced in assumption 3), $B$ an element of $\mathscr{I}$ and $\Psi$ a vector in the domain of $B$. By assumption 3 ) the automorphism $\alpha: B \rightarrow B_{\alpha}$ is weakly continuous in the group parameter $\alpha$ and commutes with Poincaré. Moreover it is locally generated by a conserved current:

$$
\frac{\partial}{\partial \alpha} B_{\alpha}=i \lim _{R \rightarrow \infty}\left[Q_{R}, B_{\alpha}\right]
$$

It is easy to see that $\forall \Phi \in G,\left\langle\Phi, B_{\alpha} \Psi\right\rangle$ is continuously differentiable in $\alpha$ and, by assump tion, periodic with period $2 \pi$. From well known results on the Fourier series

$$
\left\langle\Phi, B_{n} \Psi\right\rangle \equiv \frac{1}{2 \pi} \int_{0}^{2 \pi} d \alpha e^{-i n \alpha}\left\langle\Phi, B_{\alpha} \Psi\right\rangle
$$

exists and

$$
\left\langle\Phi, B_{\alpha} \Psi\right\rangle=\sum_{n=-\infty}^{+\infty} e^{2 n \alpha}\left\langle\Phi, B_{n} \Psi\right\rangle
$$

It is not difficult to show that, since $B_{\alpha}$ is a local field, $B_{n}$ is also local. $B_{n}$ is a field of definite charge.

5 Here and in the following $\hat{\Psi}$ will both denote the equivalence class and the vector in $H$.

${ }^{6}$ Here and in the following we assume that $H$ is separable. 
The set of vectors $\Psi_{i}$ are clearly orthonormal, by definition of scalar product in $H$ :

$$
\left(\hat{\Psi}_{i}, \hat{\Psi}_{j}\right)=\left\langle\Psi_{i}, \Psi_{j}\right\rangle=\delta_{i j} .
$$

The $\Psi_{i}$ will span a linear manifold $\mathscr{L}$ in $H^{\prime}$, whose closure $L=\overline{\mathscr{L}}$ is a Hilbert space with positive definite metric. Clearly the subspace $L \subset H^{\prime}$ is isomorphic to $H=H^{\prime} / H^{\prime \prime}$.

The definition $\hat{O}$ is now trivial. Given an operator $O$ in $G$ one firstly defines $\hat{O}$ on the orthonormal basis $\hat{\Psi}_{i}$ in the following way:

$$
\left(\hat{\Psi}_{i}, \hat{O} \hat{\Psi}_{j}\right) \equiv\left\langle\Psi_{i}, O \Psi_{j}\right\rangle
$$

and then one extends $\hat{O}$ by linearity to the whole $H$. Needless to say, different choices of vectors in the equivalence classes will give rise to different subspaces $L$, and to different definitions of $\hat{O}$. One should more pedantically write $\hat{O}^{L}$ to remind that the definition of $\hat{O}$ depends on $L$, in general. For an arbitrary operator $O$ in $G$ the mapping $O \rightarrow O \hat{O}$ is not uniquely determined.

In general the mapping ' will not preserve many of the properties of $O$. For example, in general one will have $\widehat{O}^{n} \neq \hat{O}^{n}$.

It is possible to extend the mapping ${ }^{\wedge}$ to an arbitrary vector $\Psi \in G$, by defining $\Psi \rightarrow \hat{\Psi}$, where $\forall \Psi_{i} \in L$

One has

$$
\left(\hat{\Psi}_{i}, \hat{\Psi}\right) \equiv\left\langle\Psi_{i}, \Psi\right\rangle .
$$

$$
\widehat{O \Psi_{i}}=\hat{O} \hat{\Psi}_{i} \text {. }
$$

Lemma 2. For a bounded operator $O$ defined in $G$, such that

$$
O H^{\prime} \subset H^{\prime} \text { and } O^{+} H^{\prime} \subset H^{\prime}
$$

$(+$ denotes the adjoint with respect to $\langle\cdot, \cdot\rangle)$, the mapping $\widehat{\wedge}$ is uniquely defined and one has

$$
\widehat{O^{n}}=\hat{O}^{n}, \quad \widehat{O}^{+}=\hat{O}^{*}
$$

where $*$ is the adjoint in $\mathrm{H}$.

Proof. One firstly shows that $O H^{\prime} \subset H^{\prime}$ implies $O H^{\prime \prime} \subset H^{\prime \prime}$. In fact, for any vector $\Phi \in H^{\prime \prime}$ one has

$$
\langle O \Phi, O \Phi\rangle=\left\langle\Phi, O^{+} O \Phi\right\rangle=0
$$

since $O^{+} O \Phi \in H^{\prime}$. To complete the proof one notes

$$
\begin{aligned}
\left\langle\Psi_{i}, O\left(\Psi_{j}+\Phi\right)\right\rangle & =\left\langle\Psi_{i}, O \Psi_{j}\right\rangle+\left\langle\Psi_{i}, O \Phi\right\rangle \\
& =\left\langle\Psi_{i}, O \Psi_{j}\right\rangle,
\end{aligned}
$$

$\forall \Psi_{i}, \Psi_{j} \in H^{\prime}, \Phi \in H^{\prime \prime}$, i.e. $\hat{O}$ is defined univocally. Equation (14) follows straightforwardly from (12) and (15). 
The above lemma naturally leads to consider the local * algebra $\mathscr{R} \subset \mathscr{I}$ such that $\mathscr{R} H^{\prime} \subset H^{\prime}$. (Clearly $\mathscr{R}$ contains the algebra generated by $F_{\mu \nu}$ and $j_{v}$.) The elements of $\mathscr{R}$ are good candidates to represent local observables with a physical interpretation. This is shown in the following

Theorem 3. The operator algebra $\hat{\mathscr{R}}$, defined in $H$ is a local and covariant algebra.

Proof. If $O_{1}, O_{2}$ and $O_{1}^{+}, O_{2}^{+}$leave $H^{\prime}$ invariant, for any $\Psi_{i}, \Psi_{j} \in H^{\prime}$ one has

$$
\begin{aligned}
\left(\hat{\Psi}_{i}, \widehat{O_{1} O_{2}} \hat{\Psi}_{j}\right) & =\left\langle\Psi_{i}, O_{1} O_{2} \Psi_{j}\right\rangle=\left\langle O_{1}^{+} \Psi_{i}, O_{2} \Psi_{j}\right\rangle \\
& =\left(\widehat{O_{1}^{+} \Psi_{i}}, \widehat{O_{2} \Psi_{j}}\right)=\left(\hat{O}_{1}^{*} \hat{\Psi}_{i}, \hat{O}_{2} \hat{\Psi}_{j}\right)
\end{aligned}
$$

i.e.

$$
\widehat{O}_{1} O_{2}=\hat{O}_{1} \hat{O}_{2} \text {. }
$$

Therefore one has $\left[\hat{A}_{1}, \hat{A}_{2}\right]=\widehat{\left[A_{1}, A_{2}\right]}, \forall A_{1}, A_{2} \in \mathscr{R}$ and locality is preserved by the mapping $A \rightarrow \hat{A}$.

Covariance follows from the fact that $H^{\prime}$ is Poincare invariant

$$
U(a, \Lambda) H^{\prime} \subset H^{\prime}
$$

and therefore by Eq. (16) and Lemma 2 one has

and

$$
\hat{U} \hat{U}^{*}=\hat{U} \widehat{U}^{+}=\widehat{U U^{+}}=1
$$

$$
\hat{U}^{*} \hat{U}=1
$$

so that $\hat{U}$ are unitary operators. Finally for any $A \in \mathscr{R}$, using (16) and (17), one has

$$
\hat{U} \hat{A} \hat{U}^{*}=\hat{U} \hat{A} \widehat{U^{+}}=\widehat{U A U^{+}},
$$

i.e. $\hat{A}$ has the same covariance properties as $A$.

Now we discuss the crucial point connected with the ${ }^{\wedge}$ mapping from $G$ to $H$, that is the reduction of the automorphism generated by the electromagnetic current to the operators on $H$. It is tempting to define the mapping $\hat{O} \rightarrow \hat{O}_{\alpha}$ by

$$
\alpha(\hat{O}) \equiv \widehat{\left(O_{\alpha}\right)}
$$

however we face the objection that, in general,

$$
\alpha(\widehat{A B})=\widehat{A_{\alpha} B_{\alpha}} \neq \alpha(\hat{A}) \alpha(\hat{B}) .
$$

We have proved the validity of Eq. (16) only for operators leaving invariant $H^{\prime}$ (with their conjugate). Moreover we cannot hope to generate 
locally the automorphism using a conserved current because in general the operator $\hat{O}$ on $H$ will not be local and there is no natural choice of such a current (e.g. $\hat{\mathscr{A}}_{\mu}=0$ but $\hat{j}_{\mu} \neq 0$, while in $G \mathscr{A}_{\mu}$ and $j_{\mu}$ generate the same transformation on $\mathscr{I}$ ).

It seems that the only automorphisms which can be induced in $H^{\prime}$ are those restrictions to subalgebras of $\mathscr{I}$ which are ${ }^{\wedge}$ mapped into local algebras on $H$. We have already considered an example provided by $\mathscr{R}$. From Corollary 2 we know that the elements of $\mathscr{R}$ either have zero charge or they map $H^{\prime}$ into $H^{\prime \prime}$. Going to the quotient only the elements with zero charge survive. We can then state the following theorem.

Theorem 4. If the algebra $\hat{\mathscr{R}}$ is irreducible in $H$ the automorphism of gauge transformations of the first kind is the identity and all states of $H$ have zero electric charge.

\section{Relation Between Gupta-Bleuler's and Coulomb Gauge Formulations of QED}

As discussed in the previous pages, the two formulations of QED, namely the Gupta-Bleuler's and Coulomb gauge formulations, have very different properties. The former is essentially the only way of getting a local and covariant theory of QED, whereas the latter is both non local and non covariant. From the point of view of quantum field theory they give rise to very different theories: the Wightman functions have very different mathematical properties in the two cases.

The relation between the two formulations seem, therefore, far from obvious in contrast with the statements given in the literature, where one may find "gauge transformations" leading from one theory to the other. They are formal transformations, whose mathematical meaning is not transparent. One has to recall that these transformations should destroy Lorentz covariance, locality and most likely the temperedness of the fields in Gupta-Bleuler formulation.

Here we will show that a simple way of relating the two formulations may be obtained by means of the correspondence $O \rightarrow \hat{O}$ discussed in the previous pages. As we have shown this correspondence preserves locality and covariance for the operators which leave $H^{\prime}$ invariant [like $F_{\mu \nu}(x), j^{v}(x)$ etc.] but will likely destroy both locality and covariance of the operators which lead out of $H^{\prime}$ [like $A_{\mu}(x)$ or the charged fields].

We will confine ourselves to the free field case. For the interacting case it can be easily seen that the Gupta-Bleuler formulation cannot be reduced to the Coulomb formulation by any ${ }^{\wedge}$ mapping. In fact the condition $\partial^{\mu} A_{\mu}=0$ between vectors of $H^{\prime}$ is not compatible with $\operatorname{div} \boldsymbol{A}^{c}=0, \partial^{\mu} A_{\mu}^{c} \neq 0$. 
Theorem 5. Let $A_{\mu}(x)$ denote the electromagnetic potential in the Gupta-Bleuler's formulation, i.e. $A_{\mu}(x)$ is defined as an operator valued distribution in $G$, satisfying the Wightman axioms and let $A_{\mu}^{c}(x)$ be the electromagnetic potential in the Coulomb gauge defined in a Hilbert space $H$ with positive definite metric, then, there exists a choice of $L$ (see above) such that the theories defined by $\hat{A}_{\mu}(x)$ and $A_{\mu}^{c}(x)$ are unitarily equivalent, i.e. all their Wightman functions coincide.

Proof. The vectors of $L$ are chosen in such a way that in the one particle subspace the wave functions $f_{\mu}(p)$ corresponding to them satisfy

$$
f_{0}(p)=0 \text {. }
$$

This is easily achieved, since if $\Phi_{\mu}(p)$ is a wave function describing a one particle state, $\Phi_{\mu}^{\prime}(p) \equiv \Phi_{\mu}(p)-\frac{p_{\mu}}{p_{0}} \Phi_{0}(p)$ lies in the same equivalence class as $\Phi_{\mu}$ and satisfies condition (19) ${ }^{7}$.

With this choice, it is not difficult to $\mathrm{see}^{8}$ that

$$
\left\langle\Psi_{0}, A_{0}(x) \Psi\right\rangle=0
$$

$\Psi \in L, \Psi$ being a one particle state.

Now, since $G=\bigoplus_{n=0}^{\infty}\left(H^{1}\right)^{\otimes n}$ one easily proves that

$$
\left\langle\Phi, A_{0}(x) \Psi\right\rangle=0, \quad \forall \Phi, \Psi \in L
$$

and therefore, with the above choice of $L$, one has

$$
\hat{A}_{0}(x)=0 \text {. }
$$

One has in addition

$$
\partial^{\mu}\left\langle\Psi, A_{\mu}(x) \Phi\right\rangle=0, \quad \forall \Phi, \Psi \in H^{\prime}
$$

and therefore

$$
\partial_{\mu} \hat{A}^{\mu}(x)=0
$$

Equation (20) thus yields

$$
\operatorname{div} \hat{A}=0
$$

In a similar way, after some lengthy algebra one proves that $\hat{A}_{i}(x)$ satisfy the commutation relations of the Coulomb gauge potential. Thus all the Wightman functions coincide and the two theories are unitarily equivalent.

${ }^{7}$ Note that $f_{\mu}(p)$ are zero in a neighborhood of $p_{\mu}=0$. See Ref. [7].

${ }^{8}$ For the details one has simply to use the representation of $A_{\mu}(x)$ discussed by A. S. Wightman and L. Gårding [7]. 


\section{Appendix}

In this appendix we will sketch a different proof of point (a) of Theorem 2, i.e. that $\mathscr{A}_{R} H^{\prime} \neq 0$ otherwise all the vector of $H^{\prime}$ have zero charge.

Lemma 1. If $\mathscr{A}(f) \Psi_{0}=0$ for any test function $f_{\mu}$, then the automorphism $\alpha$ induced by $j_{\mu}$ is implementable in $G$.

Proof. One first notices that $\mathscr{A}_{\mu}$ and $j_{\mu}$ generate the same automorphism on $\mathscr{I}$ so that one can consider the action of $\mathscr{A}_{\mu}$. Then for any $\phi \in \mathscr{I}$ one has

$$
\begin{aligned}
\lim _{R \rightarrow \infty}\left\langle\Psi_{0},\left[\mathscr{A}_{R}, \phi\right] \Psi_{0}\right\rangle= & \lim _{R \rightarrow \infty}\left[\left\langle\mathscr{A}_{R} \Psi_{0}, \phi \Psi_{0}\right\rangle\right. \\
& \left.-\left\langle\Psi_{0}, \phi \mathscr{A}_{R} \Psi_{0}\right\rangle\right]=0 .
\end{aligned}
$$

The irreducibility of $\mathscr{I}$ yields the implementability of $\alpha$, by standard arguments.

Lemma 2. If $\mathscr{A}(f) \Psi_{0}=0$, any vector $\Psi$ such that $\mathscr{A}(f) \Psi=0$, for any test function $f$, has zero charge.

Proof. Let $\phi_{q} \in \mathscr{I}$, be a charged field of charge $q$. Then

$$
\begin{aligned}
q\left\langle\phi_{q} \Psi_{0}, \Psi\right\rangle & =\lim _{R \rightarrow \infty}\left\langle\left[\mathscr{A}_{R}, \phi_{q}\right] \Psi_{0}, \Psi\right\rangle \\
& =\lim _{R \rightarrow \infty}\left\{\left\langle\phi_{q} \Psi_{0}, \mathscr{A}_{R} \Psi\right\rangle-\left\langle\phi_{q} \mathscr{A}_{R} \Psi_{0}, \Psi\right\rangle\right\}=0 .
\end{aligned}
$$

Thus, $\Psi$ has vanishing matrix elements with the set of charged states.

The proof of point (a) of Theorem 2, follows easily from the above lemma.

Acknowledgement. We would like to thank Professor R. Haag and Professor A. S. Wightman for their interest in this work.

One of us (F.S.) would like to thank Professor A. S. Wightman for making his stay in Princeton possible.

\section{References}

1. Gupta, S. N.: Proc. Soc. (London) 63, 681 (1950)

Bleuler, K.T.: Helv. Phys. Acta 23, 567 (1950)

2. Dirac, P.A.M.: Proc. Roy. Soc. (London) A 114, 243, 710 (1927). The principles of quantum mechanics, 4 th ed. Oxford-New York: University Press 1958; J. D. Bjorken and S. D. Drell, Relativistic quantum fields. New York: McGraw-Hill 1965

3. Streater, R., Wightman, A.S.: PCT, Spin and statistics and all that. New York: Benjamin 1964

4. Strocchi, F.: Phys. Rev. 162, 1429 (1967)

Strocchi, F.: Phys. Rev. D 2, 2334 (1970) 
5. Kastler, D., Robinson, D. W., Swieca, A.: Commun. math. Phys. 2, 108 (1966)

6. Schweber,S.S.: An introduction to relativistic quantum field theory. New York: Harper and Row 1964. For a discussion of this problem in axiomatic field theory see, F. Strocchi [4]

7. For a discussion of the properties of $H$ in the free field case see Wightman,A.S., Gårding, L. : Arkiv Fysik 28, 129 (1964)

\author{
R. Ferrari \\ Istituto Nazionale \\ di Fisica Nucleare \\ Piazza Torricelli 2 \\ I-56 100 Pisa, Italy
}

